\title{
RELAÇÃO ENTRE PRESSÃO ARTERIAL E FREQUÊNCIA CARDÍACA EM IDOSOS EM DIFERENTES TEMPERATURAS AMBIENTES
}

\author{
AUTOR: YAGO SOARES FONSECA \\ CO-AUTOR: LUCIANE APARECIDA GONCALVES MANGANELLI \\ CO-AUTOR: ALINE PRATES CORREIA \\ CO-AUTOR/ORIENTADOR: GRASIELY FACCIN BORGES
}

Resumo: Mudanças climáticas bruscas podem influenciar os ritmos biológicos, os quais interferem em todas as atividades e funções fisiológicas humanas, alterar as variáveis fisiológicas e aumentar o risco de eventos cardiovasculares, principalmente em idosos, haja vista que as ondas de calor podem exceder a capacidade adaptativa fisiológica de grupos populacionais vulneráveis. Assim, essa pesquisa objetivou analisar a relação entre pressão arterial, frequência cardíaca em diferentes temperaturas ambientes em um grupo de idosos. $O$ estudo foi feito com 72 idosos de uma associação da cidade de Teixeira de Freitas-BA, com idades acima de 60 anos e de ambos os sexos $81,9 \%$ femininos e $18 \%$ masculinos, respeitando-se os aspectos éticos como aprovação no Comitê de Ética em Pesquisa da Universidade Estadual de Feira de Santana com CAAE: 58368216.1.0000.0053. Coletou-se dados de pressão arterial sistólica, diastólica e frequência cardíaca utilizando aparelho digital da marca OMRON, modelo HEM-742INT, e relacionou-os com a temperatura ambiente e umidade relativa as quais foram coletadas com Estação Meteorológica digital Oregon Scientific, específica para áreas externas. Os parâmetros de avaliação da pressão arterial seguiram conforme as recomendações da VII Diretriz de Hipertensão Arterial (2016) e da Atualização da Diretriz de Prevenção Cardiovascular da Sociedade Brasileira de Hipertensão (2019). A análise estatística se deu por meio do teste não paramétrico de Wilcoxon, pelo software SPSS statistics. Foram comparados oito diferentes momentos(M) de acordo com as mudanças da temperatura ambiente. Verificou-se uma amplitude térmica de $9^{\circ} \mathrm{C}$ no ambiente avaliado. No grupo estudado ocorreu uma redução da pressão arterial diastólica com o aumento da temperatura ambiente $M 1\left(20,5^{\circ} \mathrm{C}\right)=133,2 \mathrm{mmHg}$ e $M 7\left(28^{\circ} \mathrm{C}\right)=131,0 \mathrm{mmHg}$ com $\mathrm{p}=0,018(\mathrm{p}<0,05)$, os demais não mostraram diferenças significativas. As mudanças climáticas, sobretudo os aumentos ou diminuições bruscas de temperatura, estão relacionados ao desenvolvimento e agravo de doenças cardiovasculares por todo o mundo, dentre elas a hipertensão. Entretanto em ambientes com baixa variação térmica não foi observada influência significativa sobre a pressão arterial e frequência cardíaca, sendo necessárias mudanças mais bruscas para que isso ocorra nos idosos avaliados.

Palavras-chave: Pressão Arterial, Hipertensão, Temperatura Ambiente, Idosos. 